\title{
Jurnal Geofisika Eksplorasi (JGE)
}

JGE (Jurnal Geofisika Eksplorasi) - Journal of Exploration Geophysics - is a doubleblind peer-reviewed journal that is managed and published by Geophysical Engineering Department, Engineering Faculty, Universitas Lampung. JGE is published periodically (three times a year) in March, July and November. JGE is available for free (open access) to all readers. The articles in JGE include developments and researches in Geophysical Exploration and Earth Science (theoretical studies, experiments, and its applications).

\author{
Person in Charge \\ Head of Geophysical Engineering Department \\ Engineering Faculty \\ Universitas Lampung \\ Editor in Chief \\ Rahmat Catur Wibowo S.T., M.Eng.
}

\section{Editorial Advisory Board}

Prof. Dr. Andri Dian Nugraha, M.Si., Geophysical Engineering, ITB, INA

Dr. Ir. Nandi Haerudin, M.Si., Geophysical Engineering, Universitas Lampung, INA

Dr. Ir. Muh. Sarkowi, S.Si., M.Si., Geophysical Engineering, Universitas Lampung, INA Dr. Asep Harja, Geophysics, Universitas Padjajaran, INA

Dr. Ir. D. Hendra Amijaya, M.T., Geological Engineering, UGM, INA

Dr. Ir. Ferian Anggara, M.T., Geological Engineering, UGM, INA

Indra Arifianto, S.T., M.Eng., Computational Earthquake Seismology, KAUST, SA

\section{Editorial Member}

Sandri Erfani, S.Si., M.Eng., Geophysical Engineering, Universitas Lampung, INA I B Suananda Yogi, S.T., M.T., Geophysical Engineering, Universitas Lampung, INA Rudy Z Sinambela, S.T., M.T., Geophysical Engineering, Universitas Lampung, INA

Isti Nur Kumalasari, S.T., M.T., Oil and Gas Engineering, ITERA, INA

Istifani Ferucha, S.T., Geophysical Engineering, Universitas Lampung, INA

\section{Postal Address}

Jurusan Teknik Geofisika, Fakultas Teknik Universitas Lampung

Jl. Prof. Dr. Sumantri Brojonegoro No.1 Bandar Lampung

Telp. (0724)704947 Fax. (0721)704947

Email: jge.tgu@eng.unila.ac.id

Website: https://jge.eng.unila.ac.id/index.php/geoph 


\section{JGE Peer-Reviewer}

Prof. Dr. Ir. Hendarmawan, M.Sc., Universitas Padjajaran, Indonesia

Prof. Suharso, P.hD., Universitas Lampung, Indonesia

Prof. Dr. Ir. Binarko Santoso, R\&D Centre for Mineral and Coal Technology, Indonesia

Prof. Dr. Drs. Sismanto, M.Si., Universitas Gadjah Mada, Indonesia

Prof. Sri Widyantoro, M.Sc., P.hD., Institut Teknologi Bandung, Indonesia

Prof. Adi Susilo, Universitas Brawijaya, Indonesia

Prof. Ir. Lambok Hutasoit, M.Sc., P.hD., Institut Teknologi Bandung, Indonesia

Prof. Dr. Supriyadi, Universitas Negeri Semarang, Indonesia

M. Yusuf Nur Khakim, Ph.D., Universitas Sriwijaya, Indonesia

Dr. Muksin Umar, Universitas Syiah Kuala, Indonesia

Dr. Tedi Yudistira, Institut Teknologi Bandung, Indonesia

Dr.Eng. Supriyanto Suparno, M.Sc., Universitas Indonesia, Indonesia

Dr.Eng. Imam Achmad Sadisun, Institut Teknologi Bandung, Indonesia

Dr.Eng. Suryantini, M.Sc., Institut Teknologi Bandung, Indonesia

Dr. Maman Hermana, Universiti Teknologi Petronas, Malaysia

Dr. S.S. Rita Susilawati, Coal and Geothermal Mineral Resources Center, Indonesia

Dr. Sri Hidayati, Volcanology, Mitigation and Geological Disasters Center, Indonesia

Dr. Eko Januari Wahyudi, Institut Teknologi Bandung, Indonesia

Dr.Eng. Asep Saepuloh, Institut Teknologi Bandung, Indonesia

Dr.rer.nat. Wiwit Suryanto, S.Si., M.Si., Universitas Gadjah Mada, Indonesia

Dr. Arif Ismul Hadi, M.Si., Universitas Bengkulu, Indonesia

Dr. Eng. Yunus Daud, Dipl.Geotherm.Tech., M.Sc., Universitas Indonesia, Indonesia

Dr. Syukri Surbakti, Universitas Syiah Kuala, Indonesia

Ir. Dida Kusnida, M.Sc., R\&D Centre for Marine Geology, Indonesia

Imam Setiadi, S.Si., M.T., R\&D Centre for Marine Geology, Indonesia

Drs. Eddy Supriyana, M.Si., Universitas Padjajaran, Indonesia

Herning Dyah Kusuma W., M.Eng., Institut Teknologi Nasional Yogyakarta, Indonesia

Rahmadi Hidayat, S.T., M.Eng., University of Wollongong, Australia

Tri Winarno, S.T., M.Eng., Universitas Diponegoro, Indonesia

M. Haris Miftakhul Fajar, S.T., M.Eng., Institut Teknologi Sepuluh Nopember, Indonesia

M. Firman Gazhali, S.Pd., M.T., Universitas Lampung, Indonesia

Normansyah, S.Si., M.Si., PT. PHE ONWJ, Indonesia

Rian Amukti, S.Si., M.Sc., LIPI, Indonesia 


\section{T: Jurnal}

Jurnal Geofisika Eksplorasi (JGE)

\section{TABLE OF CONTENTS}

88 Inversi Data Geolistrik Menggunakan Particle Swarm Optimization: Studi Kasus Desa Gayau, A. Farduwin, R.M. Antosia, I.A. Putri, N.A. Santoso, S.M. Irawati

100 Investigasi Air Tanah Berdasarkan Nilai Resistivitas di Dusun Jatisari, Kabupaten Lampung Selatan, A.Y. Paembonan, P. Nugraha, N.A. Santoso, R. Firdaus, G.M. Ekawati, V. Rahmanda, F.D.P. Amijaya

111 Geologi Gunungapi Purba Soreang dan Implikasinya Terhadap Potensi Cebakan Emas, R. Ikhram, I. Arifin, R.I. Sophian

126 Geothermal Potential on Sumatra Fault System to Sustainable Geotourism in West Sumatra, H.W. Utama, R. Mulyasari

138 Alterasi dan Mineralisasi Bijih Pada Batuan Diorit di Daerah Wombo, Sulawesi Tengah, A.N.N. Rahmaningrum, Asrafil

150 Analisis Karakteristik Lapisan Sedimen Berdasarkan Data Mikrotremor di Area Rumah Sakit Pendidikan Unila, Hesti, Suharno, R. Mulyasari, A. Hidayatika 